\title{
Connection of large earthquakes occurring moment with the movement of the Sun and the Moon and with the Earth crust tectonic stress character
}

\author{
M. K. Kachakhidze ${ }^{1}$, R. Kiladze ${ }^{2}$, N. Kachakhidze ${ }^{1}$, V. Kukhianidze ${ }^{2}$, and G. Ramishvili ${ }^{2}$ \\ ${ }^{1}$ Department of Natural Sciences, St. Andrew The First-Called Georgian University of The Patriarchy of Georgia, \\ Tbilisi, Georgia \\ ${ }^{2}$ Department of Natural Sciences, Ilia State University, Abastumani Astrophysical Observatory, Tbilisi, Georgia
}

Received: 2 February 2010 - Revised: 30 May 2010 - Accepted: 13 July 2010 - Published: 29 July 2010

\begin{abstract}
It is acceptable that earthquakes certain exogenous (cosmic) triggering factors may exist in every seismoactive (s/a) region and in Caucasus among them. They have to correct earthquake occurring moment or play the triggering role in case when the region is at the limit of the critical value of the geological medium of course.

Our aim is to reveal some exogenous factors possible to initiate earthquakes, on example of Caucasus s/a region, taking into account that the region is very complex by the point of view of the tectonic stress distribution.

The compression stress directed from North to South (and vice versa) and the spread stress directed from East to West (and vice versa) are the main stresses acted in Caucasus region. No doubt that action of the smallest external stress may "work" as earthquakes triggering factor.

In the presented work the Moon and the Sun perturbations are revealed as initiative agents of earthquakes when the directions of corresponding exogenous forces coincide with the directions of the compression stress or the spreading tectonic stress in the region.
\end{abstract}

\section{Introduction}

It is necessary to assume that if it is not the variable stress in the earth (inter earth, meteorological or space origin) the earthquake will occur at the moment, when tectonic stress in the zone of its preparation reaches the certain critical meaning - the limit of the geological medium strength.

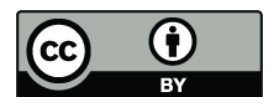

Correspondence to: M. K. Kachakhidze (manana_k@hotmail.com)
The presence of the variable stress in the Earth considerably changes this situation. These stresses being added on the smooth increasing tectonic stress can prematurely bring a total stress up to critical meaning and thus provoke earthquake at a rather early stage of its preparation. From this point of view we may think that earthquakes are not "ripen" up to the end and do not occur themselves. The moment of the earthquakes occurring depends on the joint action of the factors causing the variable stress in the Earth.

By their nature these initiative factors can be divided into two kinds - internal (endogenous) and external (exogenous).

It is acceptable that internal forces oriented along tectonic stresses may have the most important influence on the seismicity of region. In order to check this suggestion we studied this work.

Many scientific papers are devoted to the problem of the role of the cosmic factors in the earthquakes occurring process (Harris, 2000; Lopes et al, 1990; Nikolaev, 1995; Nikolaev et al, 1996; Rydelek et al, 1992; Tanaka et al, 2006; Tanaka, 2007; Tsuruoka et al, 1995; Van Ruymbeke et al, 2007). In some cases there are shown the Sun perturbations and in other cases - the Moon perturbation components influence on the earthquakes occurring moments. There are papers where the Sun and the Moon perturbations influence on the earthquakes are not fixed at all (Davidson, 2002; Davidson, 2005).

The task of determination of the exogenous action on the Earth from the main celestial bodies must be solved for any point and any moment of the time. The fact that tides and deformations are depended on the geographic coordinates must be taken into account (Kiladze et al, 1997, 2005).

Published by Copernicus Publications on behalf of the European Geosciences Union. 


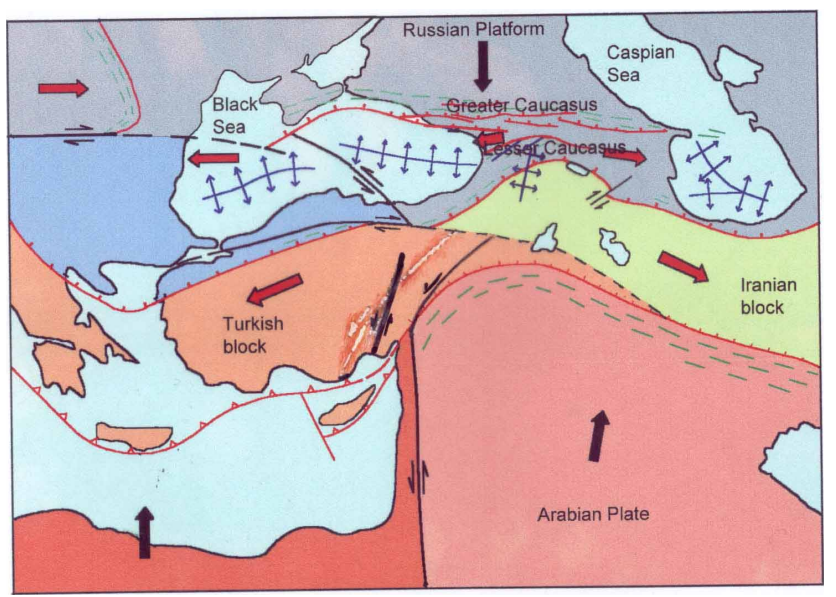

Fig. 1. Tectonic stress map of Caucasus and neighbouring regions. Large red arrows are the approximate directions of motion of different parts of Caucasus and neighbouring regions relative to Eurasia; the black arrows correspond to the compression fields.

We must note that the task of determination of direction of tectonic stress action is solved with less accuracy. The field of the tectonic stress has the hierarchical structure.

As it is known the different total tectonic stress acts in the different regions because of plates movements particularities.

It is established that in the young folded areas like the Caucasus the field of tectonic stress is characterized by the sharp anisotropy with the predominance of the compression perpendicular to the trend of folding. Spatial location of the main positive and negative geotectonic morphostructures of the Caucasus shows the existence of the wavy tectonic movements in the region. They are caused by the horizontal compression, provoked evidently by advancement of the Arabian lithosphere plate to the North and its reaproachment with the Euroasian plate (Fig. 1). All these cause considerable deformation of the lithosphere of the Caucasian region and it is breaking up in separate blocks. This, in its turn, causes the concentration of stress along the boundaries of the blocks and rising of earthquakes focuses there (Jackson, 1992; Kachakhidze et al., 2001).

Basing on the geological and geophysical data we can pick out in Caucasus:

1. Interzonal deep faults of the Caucasian orientation, which divide the main tectonic units of the region.

2. Interzonal longitudinal and transversal faults.

3. Transzonal faults of the Anticaucasian and submeridional trend.

At present these faults determine heterogeneity of the Caucasian stress field, which is mainly characterized by the shear stress, directed diagonally towards the main structures and finally-by the tensile stress, directed subaltitudinally, parallel with the main structures.
As we mentioned above, in every region, including the Caucasus, besides of geological factors it may be the exogenous (cosmic) force of certain direction, which takes role of triggering factor in the occurring process of large earthquakes, if tectonic stress is near to its critical value.

It is known that any planet is not only under the Sun attraction but under other bodies of the Solar system also. As the planets of the Solar system have the mass many times less than the Sun, the Sun and the Moon are considered as the "main bodies" of perturbation.

Thus, the stress acted on the Earth from the solar system and, in our case, on the Caucasian region, can play a role of the triggering factor only in case when its direction helps to increase tectonic stress. At the same time this additional stress acts on the neighboring region of course (for instance on Turkey and Arabian Peninsula) but tectonic stress has different direction there. Because of it the force acted on the Caucasus region, from the Solar system bodies, can not play the role of the triggering factor for Turkey or Arabian Peninsula. On the contrary, the exogenous stress added to the Turkey tectonic stress appears as earthquakes initiative factor for Turkey and can not play the role of the triggering effect for the Caucasus and Arabian Peninsula earthquakes.

\section{The method}

Unfortunately, it is not possible to determine value of the force caused by plate's movement and the task of $\mathrm{N}$ - body is not solved analytically yet.

Consequently, we are made to investigate earthquakes exogenous triggering factors by numerical methods.

We consider earthquakes occurring moments connection with the Sun, the Moon attractive and gravity perturbation components of tide which will be called in future as "initiative agents" of the earthquakes.

Vertical $(Z)$ and horizontal $(D)$ components of an attraction are following:

$$
\begin{aligned}
& F_{Z}=G \frac{m M}{R^{2}} \cos Z \\
& F_{D}=G \frac{m M}{R^{2}} \sin Z \cos (A-D)
\end{aligned}
$$

where $G$ - is gravitational constant, $R$ - distance up to the celestial body, $Z$ and $A$ - its zenith distance and azimuth and $D$ - azimuth of a horizontal projection of the force.

Zenith distance and azimuth of the celestial body are calculated by the formulas:

$\cos Z=\sin \phi \sin \delta+\cos \phi \cos \delta \cos t$

$\tan A=\frac{\cos \delta \sin t}{\sin \delta \cos \phi-\cos \delta \sin \phi \cos t}$

where $\phi$-is latitude of the terrain, $\delta$ - declination of celestial body and $t-$ its hour angle. 
Table 1. Azimuths of the perturbation forces.

\begin{tabular}{lccc}
\hline Triggering factor & Azimuth $A$ of the force direction & $\frac{n-\bar{n}}{\sigma}$ & Validity of the phenomena \\
\hline Perturbation from the Sun & $193^{\circ}-206^{\circ}$ & 2.35 & $98 \%$ \\
Perturbation from the Moon & $295^{\circ}-310^{\circ}$ & 3.28 & $99.8 \%$ \\
Total perturbation & $290^{\circ}-307^{\circ}$ & 1.72 & $91.5 \%$ \\
\hline
\end{tabular}

Tides caused by the celestial body, are calculated by the formulas:

$\Delta F_{z}=\frac{G r m M}{R^{3}}\left(1-3 \cos ^{2} Z\right)$

$\Delta F_{D}=-3 \frac{G r m M}{R^{3}} \sin Z \cos Z \cos (A-D)$

where $r-$ is the radius of the Earth.

\section{Discussion}

We considered all 393 Caucasus earthquakes with $M \geq 4.5$. Histogram of earthquakes recurrence frequencies were constructed based on these observed frequencies at different intervals of azimuths (we considered frequencies of recurrence of azimuths in intervals of tides possible directions). The interval of possible azimuths was divided into 18 parts. In addition to it three histograms are created for the Sun, the Moon tides and for their total perturbations (Fig. 2a, b, and c).

In Table 1 there are shown azimuths of the force directions (for the Sun, the Moon and total) deviation from the average meaning of which is more then $2 \sigma$.

There are given: triggering factor, the meanings of the azimuths of the celestial bodies at the moment of the occurring of the significant quantity of the earthquakes, the relative detour from the average meaning and validity of the cases. Such four directions are revealed in all. In these cases the quantity of the earthquakes are more than their average significances.

Sectorial effect is obviously shown in the Moon gravity perturbations and in the total gravity perturbations of the Sun and the Moon (Fig. 2b and c).

The Moon gravity perturbation effect is given in the Fig. 2 . It is shown the heterogeneity in the azimuth sector $295^{\circ}-$ $310^{\circ}$, but the total perturbation effect - in the sector $290^{\circ}-$ $307^{\circ}$ (Fig. 2c). It is obvious the great share of the Moon in the total effect.

It is clear that the each possible "agent" has only one azimuth, to which the increased quantity of earthquakes corresponds (Table 1).

In order to more detail analysis of task the Moon, the Sun and their total perturbation directions were calculated and inflicted on the Caucasus main faults map for each earthquake separately. Only three of them are shown in the Fig. 3 like examples (Fig. 3a, b, and c).

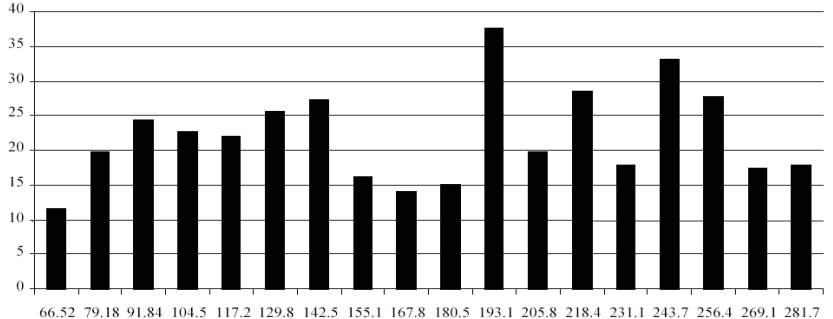

(a)

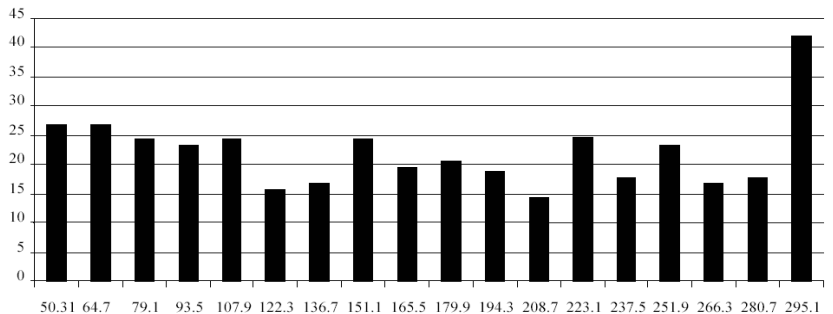

(b)

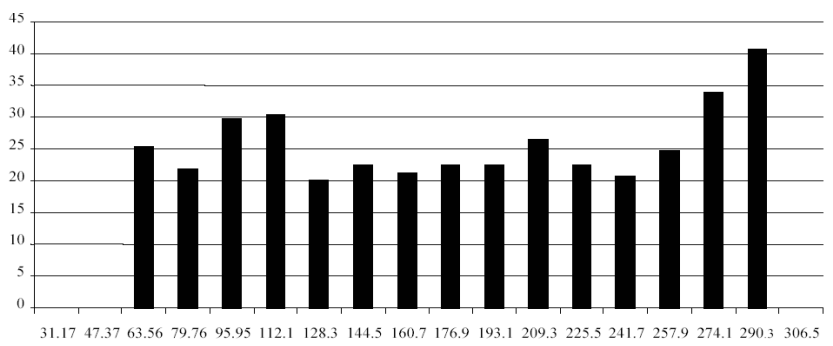

(c)

Fig. 2. Histograms: (a) Sun gravity perturbation; (b) Moon gravity perturbation; (c) the total (the Sun and the Moon) gravity perturbation.

\section{Conclusions}

By comparing of getting results with Caucasus tectonic map we may do conclusion that the majority quantity of Caucasus $M \geq 4.5$ earthquakes occur when:

1. Gravitational field and the Sun perturbation are perpendicular to the plane of Russian platform (i.e., they are perpendicular to fault along Caucasian mountain ridge) and they are directed from the North to the South, in particular, create $20^{\circ}-30^{\circ}$ angles with $40^{\circ}$ meridian. 
(a)
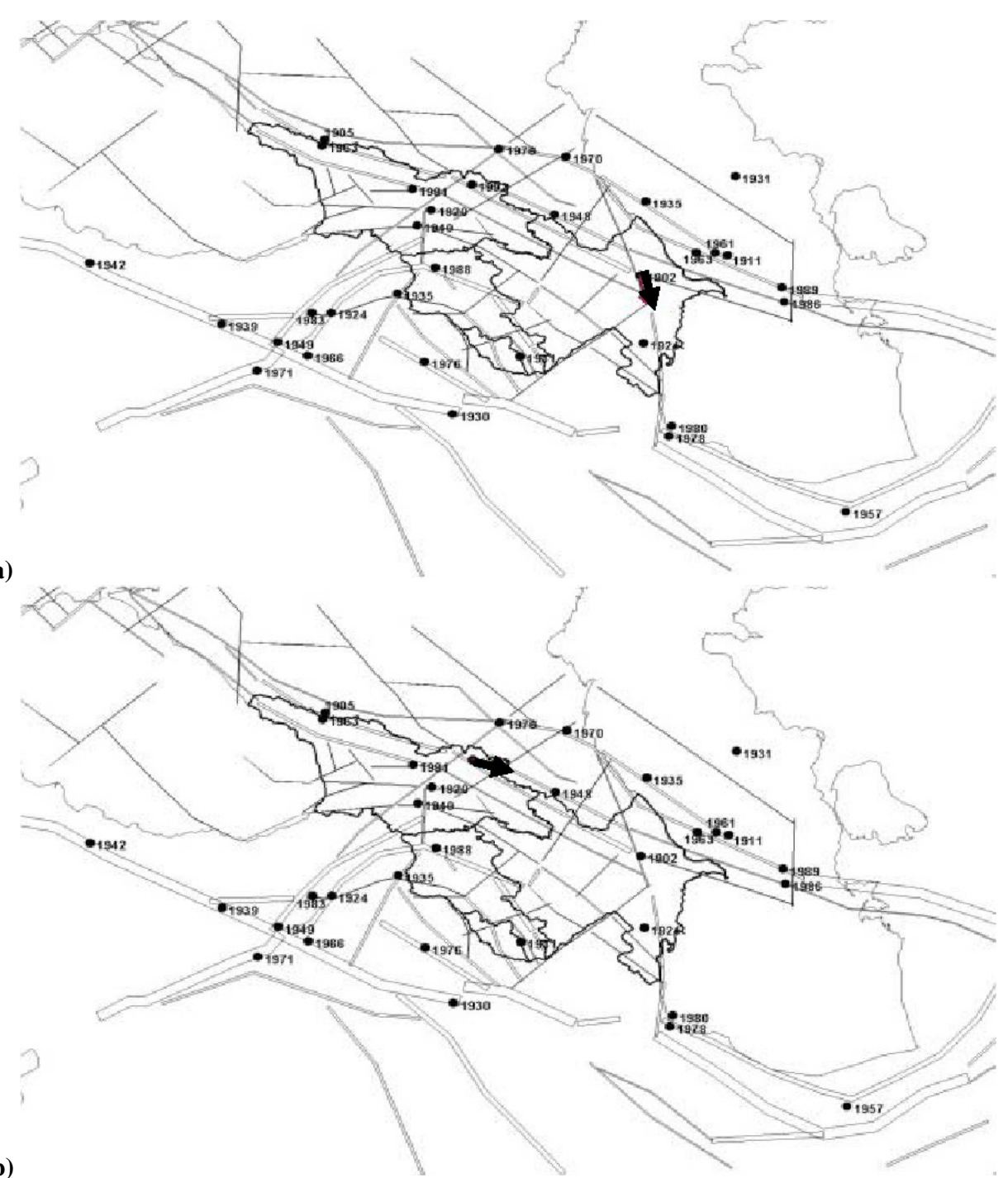

(b)

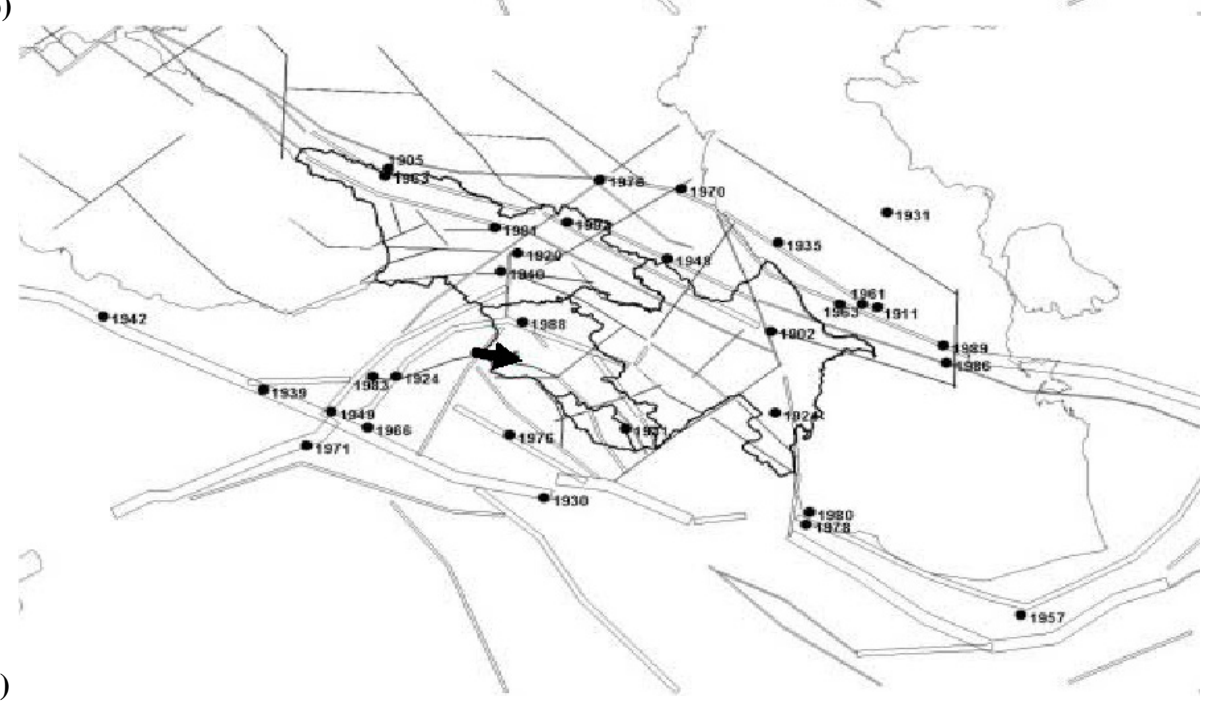

Fig. 3. Arrows: (a) the Moon tide direction at Caucasus 1902 earthquake occurring moment; (b) the Sun tide direction at Caucasus 1992 earthquake occurring moment; (c) total tide direction at Caucasus 1935 earthquake occurring moment. 
2. Lunar and total perturbations coincide with the direction of the tectonic stress, approximately, from SouthEast to North-West (along the above mentioned fault in the Caucasian seismoactive region) and is known as the stretching stress. They create $60^{\circ}$ angle with $40^{\circ}$ meridian.

Thus, based on Caucasus region earthquakes, we conclude that, in case of consideration of the regional tectonic stress picture, we must take into account above described distributions of the celestial bodies on the heaven and relevant time intervals as the periods of increasing danger of large earthquakes occurring in the region.

Edited by: M. E. Contadakis

Reviewed by: P. F. Biagi and another anonymous referee

\section{References}

Davidson, S.: Solar and Lunar Triggers on Earthquakes and Volcanic Eruptions, Gravity Concepts, Sec. 70, 175-181, 2 March 2002.

Davidson, S.: High Tides Trigger Earthquakes, Gravity Concepts, Sec. 30, Rev. 1, 2005.

Harris, R. A.: Earthquake stress triggers, stress shadows, and seismic hazard, in: Special Section: Seismology, Current Science, 79(9), 1215-1225, 2000.

Jackson, J.: Partitioning of Strike - Slip and Convergent Motion Between Eurasia and Arabia in Eastern Turkey and the Caucasus, J. Geophys. Res., 97(89), 12.471-12.473, 10 August 1992.

Kachakhidze, M., Kachakhidze, N., Kiladze, R., Kukhianidze, V., and Ramishvili, G.: Tectonic sources of caucasus strong earthquakes, Nat. Hazards Earth Syst. Sci., 4, 53-58, doi:10.5194/nhess-4-53-2004, 2004.

Kiladze, R., Kachakhidze, M., Kukhianidze, V., Kachakhidze, N., and Ramishvili, G.: Study of possible relationships between strong earthquakes and astronomical phenomena for seismoactive regions of the Caucasus, Turkey and Greece, Bulletin of Georgian Academy of Sciences, 155(N3), 367-369, 1997.
Kiladze, R., Kachakhidze, M., Kukhianidze, V., Kachakhidze, N., and Ramishvili, G.: A Search of Possible Relations Between Large Earthquakes and Astronomic Phenomena: the Caucasian Seismic region, J. Vulkanol. Seismol., (N3), 75-80, 2005.

Lopes, R. M. C., Malin, S. R., Mazzarella, A., and Palumbo, A.: Lunar and solar triggering of earthquakes, Phys. Earth. Planet. In., 59, 127-129, 1990.

Nikolaev, A. V.: On the earthquake triggering by the Earth's tide and underground nuclear explosions, in: Earthquakes induced by underground nuclear explosions, NATO ASI Series, 2-Environment, 255-266, 1995.

Nikolaev, A. V. and Nikolaev, V. A.: Lithosperic stress state in South Americans inferred from tidal triggering of erthquakes, Geophys. J. Int., 35(N3), 329-339, 1996.

Rydelek, P. A., Sacks, I. S., and Scarpa, R.: On tidal triggering of earthquakes at Campi Flegrei, Italy, Geophys. J. Int., 109, 125137, 1992.

Tanaka, S., Sato, H., Matsumura, S., and Ohtake, M.: Tidal triggering of earthquakes in the subducting Philippine Sea plate beneath the locked zone of the plate interface in the Tokai region, Japan, Tectonophysics, 417, 69-80, 2006.

Tanaka, S.: Tidal triggering of earthquakes precursory to the recent Sumatra megathrust earthquakes of 26 December $2004\left(M_{w} 9.0\right)$, 28 March $2005\left(M_{w} 8.6\right)$, and 12 September 2007( $\left.M_{w} 8.5\right)$, Geophys. Res. Lett., 37(2), L02301, doi:10.1029/2009GL041581, 2010.

Tsuruoka, H., Ohtake, M., and Sato, H.: Statistical test of the tidal triggering of earthquakes: contribution of the ocean tide loading effect, Geophys. J. Int., 122, 183-194, 1995.

van Ruymbeke, M., Zhu, P., Cadicheanu, N., and Naslin, S.: Very Weak Signals (VWS) detected by stacking method according to different astronomical periodicities (HiCum), Nat. Hazards Earth Syst. Sci., 7, 651-656, doi:10.5194/nhess-7-651-2007, 2007. 